\title{
Investigating BI in Real Time Systems: Concepts, Components, Techniques and Benefits
}

\author{
Zafar Ali Khan and M.N.A. Khan \\ Shaheed Zulfikar Ali Bhutto Institute of Science and Technology, Islamabad, \\ Pakistan \\ zafar786786@gmail.com,mnak2010@gmail.com
}

\begin{abstract}
The automated systems that support in decision making process are known as Business Intelligence Systems. In the past 10 years, the importance of Business Intelligence Systems has gained widespread acceptance by the industry. There are a large number of organizations that are now dealing with great number customers and enormous amount of data. To meet their growing computation needs and enhance their timely decision making capability, these organizations are highly relying on business intelligence applications to meet the challenges posed by the today's competitive market. To overcome these challenges, the companies and organizations integrate enterprise applications with the real time systems such as decision support systems and business intelligence systems. There are several companies and organizations that are using Business Intelligence tools to get knowledge from the history data and to make plan and educated guesses for the future trends and demands. Many companies know the importance and role of Business Intelligence to make effective and timely decisions in order to enhance their productivity and keep abreast with the new market demands. Due to the recent technological advancements and availability of state-of-the-art software tools, the Business Intelligence Systems have gained significant importance in the business world. Currently, most of the organizations around the globe are adopting Business Intelligence solutions to earn maximum profits, reduce recurring expenditures, promote business and retain customers. Business Intelligence is defined as an organization's capability to gather all its strengths and skill sets and transform theses into a knowledge which is now known as a currency of the business. The acquired information or knowledge may be linked to the large amounts of historical data which may lead to new vistas of opportunities for the organization. In this paper, we present a comparative study of the business intelligence tools and techniques used to make effective decisions.
\end{abstract}

Keywords: Business Intelligence, BI Concepts, BI Tools, Knowledge Management

\section{Introduction}

Business Intelligence is used by the organizations to analyze the data and on the basis of this data, better decision can be made. It is need of the time that organizations review their business requirements to implement Business Intelligence tools for better decision making. But there are still many organizations which have some challenges to become part of the BI community, and some have implemented $\mathrm{BI}$ application but they are unable to get maximum benefits from the BI solutions because they are not familiar with the concepts of BI and are not fully ready for BI. Enterprise managers take important decision on the basis of data provided by the BI tools, so nowadays no one can ignore the importance of BI and BI tools in the market. Business Intelligence technologies provide the activities of decision support systems. MIS systems and executive information systems. 


\section{Literature Review}

Ciobaju et. al., [1] described BI synchronization on distributed environment and provide solution through network for internet servers. In this paper he proposed a common solution for all BI application that runs on different servers on the internet. For his goal he did a case study on Microsoft BizTalk server as a BI system and called this research a PrO Processor. He did this research because there was a need of that kind of research through which we can communicate on different servers with the help of internet where BI applications are installed. In this research he provides a common synchronization architecture that is intra coordination among different BI application installed on different server. The research for configuration of BI application installed on their servers. They also suggest the security of the communication by using TLS and SSL protocols. The system also gave facility for tracking records of all clients. The servers maximize the number of client demands and minimize the server hardware requirement. In the future we can add more value in this by adding the bigger BI solutions such as Oracle, SAP etc., we can also go for the different versions of the same Research. In the research, Pro processor software is introduced. Pro-processor is software that is used for clear; it is used because the companies need system. The Pro processor generates a report that is legal document used by the public institutions. The main module of the Pro Processor are; configuration module that is responsible for configuration of the master data, the data acquisition module, that create link for the Pro processor to communicate with other local system of the host company, the data processing module, that create reports on the requirement gathered, the eSafe that take the responsibility of security issues and finally the report dispatching module that generates the report for public institutions.

The architecture of Pro-system is also introduced, and it gets the data from the customer into the internal system. During the implementation the first step was the synchronization of BI system with source and the target data. The system was implemented with the help of BI application and synchronization packages were installed. The research paper introduced multiple business intelligent system to be synchronized via internet. The research also helps by giving chance to small scale businesses that do not have sufficient fund for BI applications. The research further opens ways for the synchronization of BI system.

Houxin You [2] defined some fact about Real-time BI. He explains that the tradition and conventional BI which is used by organizations for meaning full information that support in decision making and to check customer loyalty and retentions with the organization is now changed. Due to the globalization the trend is going to change and there are lots of choices in the market. Now the firms are thinking for some change and they are going for real time BI to solve their problems. In this paper he tried to overcome the disadvantages by deploying novel model of knowledge management for data mining process to reduce data, its analysis and to reduce the latency for real time BI. The first step begins with hypothesis to conceptualize the knowledge management for data mining. Secondly the data mining results are summarizing for data mining process which produces a significant level of conclusion. Thirdly the decision is made on the data provided by data mining process. Fourthly the business decision is evaluated and its execution is verified in terms of tangible and intangible benefits and costs. The paper explain new novel model for Knowledge management through data mining to get the maximum benefit for industry. It's a new research area so its needs further studies.

Ren [3] explained the importance of SAP in BI applications. SAP BI is one of the most famous enterprise BI applications which is used to help in decision making. It plays am vital role to keep the product and services up to the mark and compete the organization. In this paper Ren explain the importance, components. He also 
describes. This paper helps in analysis, for the organization. The SAP BI application, very important BI tool which provides maximum support for decision making. It is also used for external data source to be load, transport and merge in B I tools. The tool also provides reporting, analysis and planning for decision making on basis of data provided. In the paper BI is used in SAP NetWeaver. The paper explained the. The paper also explained the feature of technologies used in this paper. The tools provide that allow access to multiple portal. In this research Ren explained all the components in detail and their importance in the BI system. Some more features are; there is a new object concept for the DataSource. Options for direct access to data have been enhanced. From BI, remote activation of DataSources is possible in SAP source systems. The data transfer process distributes the data within BI. It replaces the InfoPackage for updating data from the PSA, and the Data Mart Interface for updating data from one InfoProvider to another. Finally in this paper the importance of SAP BI is clearly described in decision making.

Hussain et. al., [4] define Business Intelligence importance and security concern in an organization. They explain that BI is very critical software deployed by any organization to extract data and reports through Online Analytical Processing to take higher level decisions. In this paper he explains and suggests the security issue of cloud based BI which is off high importance issue for the higher management. Because any user can take access on the very importance and sensitive data of an organization. They suggest the BI environment should be protected through database objects, application files and the underlying servers. In this paper they simulate two models to secure BI on the clouds. The first method is Unified Thread Management (UTM-security as service) and the second is distributed security model embedded in BI server arrays installed through the cloud. During the simulation it should that the first model is expected more threat and drawback while the second one is showing better result than the first model for security, and it is better used for administrative purposes. But by using a mixed approach of both the model provide maximum security, better security process and clear and effective accountability. Cloud computing has a high level importance in computing power. This research provides opportunities for BI to handle big data and also provide future direction for further research. The Model described three large model for BI cloud computing; an extranet domain of BI user, the UTM model and cloud hosing BI services. The BI cloud consists of two server arrays which is commonly used for UTM and embedded security model. The DW_DM server that is used for data warehouse and data mart servers hosting the temporal and non temporal database. The OLAP app server that take the responsibility of OLAP dashboard applications. The servers are connected through the array of cloud switched managed by with the help of Cisco 7000 series switches. In this way the model is designed and implemented and tested. The average response to database queries on the network was between 20 to 40 seconds.

The first model that comprising access of BI user a UTM cloud offering security as a service. The second model with UTM cloud eliminated and all users directly connected to BI application server on the cloud. This is the system which is proposed in the paper for high level of security and accountability for concurrent user access. Cloud computing is new emerging technology in IT industry and provide significant computing power and storage capacity for the data warehouse. That provides many complex domains for BI which is impossible for self hosted environment. Application like context-aware, location aware automation, massive scale semantics, advance science and technology databases, real time crises and disaster management, city management, global financing and economy reporting and global monitoring of industry are few area where cloud computing and BI provide tremendous opportunities. It needs research and further studies. Wang et. al., [5], defined the importance of Cloud Computing and Business Intelligence 
together in this research paper. He explains the technologies and architecture of cloud computing and BI. It is also compared with tradition BI and examines the advantage and feature of cloud computing based BI. In the research paper first explain the complete architecture of BI and describe its components. The paper described the BI architecture and explains it major components. The architecture is. The whole system is scheduled for Meta data management. To compare traditional BI and the could based BI they explain the tradition BI and showed graphically that there are four categories namely Front End Application, Data Storage, Data integrity, Data Resources. The present the physical architecture of Cloud Computing based BI. The tradition BI system has mature process link reporting tools, which has their advantage and disadvantages. Whereas, the cloud computing based BI has much advantage. One of the most developing directions for BI is BI with Cloud Computing. In future enterprise will focus on it and will give much investment for development. The paper explains the importance of cloud computing in BI marketplace. Due to data capacity the enterprises are moving toward Cloud base BI and investing more in this field. In this way the organization will be able to meet the new challenges and bitterly analy sis the large scale data. The paper gives us future direction in this field.

Dayal et. al., [6], explained the data flow, technologies and tools of BI. The explains that BI is used several operation databases and facilitates number Data warehousing is gathering by on way process. This done in offline mode so decision making during this time cannot happened. The paper explains that design and implementation of data ware housing is now a labour work. They explain that the enterprises are becoming more automated and they need quick decision making on their data to get maximum benefit and overcome their competitors. For this purpose the paper explain the importance of Real Tim e Business Intelligence. It will reduce the data latency and data will be provided to the data warehouse. This also needs some modification in data warehousing and technical support. Dayal et. al., [6] explained traditional Business Intelligence architecture and the next generation business intelligence architecture and integrate the data integration flow in the future BI. He also explained why it is required and its benefits. With increasing demand of data warehousing ETL design has become more complex so there is a need to design such system having data integration facility. He also explained the next generation data integration flow in detail. He described an example scenario that was used throughout the research. The enterprise database is based on the structure data but in future we expect that the enterprise data will have semistructure and unstructured data for its data warehouse. In the current system the ETL process is on way, from operation data to the enterprise data warehouse. In future it may support some more functionality. The outcome of data cleaning could be part of the operation system to improve accuracy and reduce cleansing work. The paper explains challenges, design and implementation of optimal flow for the real time BI. The paper gives future directions and explains the drawback of current data warehouse process. In the paper the importance of automated process of data warehousing is described because it will remove the labour work. It also explains that there is need for optimization the process because in future we need the real time data to be part of the data warehouse.

Cvitas [7] explained importance of using Business Intelligence solutions in the enterprises. He explains that BI plays a vital role in quick decision making by analyzing data provided by data warehouse. The BI decision making is dependent on the source data. The paper explains alternative way of using strictly structured data. The paper gives an overview of many proposed techniques for information extraction. Cvitas [7] explained information extraction and retrieval, information extraction types, machine learning, machine learning methods for information 
extraction and information extraction in BI. He explains that BI is set of application and technologies that is used for gathering, storing, accessing and analyzing data for decision making. The BI applications are dependent on data warehouse and data warehouse is responsible to provide clean and standard data while the data warehousing is depending on the mature process of ETL to When there is more complex analysis and reports, BI needs import of data. If the data is structured then ETL process is used. But when the data is unstructured then the textual format document is used rather than ETL as described in the Fig. Not all types of information are relevant and can be used in BI. In the paper information extraction from the BI system described in detail. He explained that information extraction can play two different roles depending on the data or text it processes. The first step can give you complete knowledge which can be used for the decision making while in the second role the set of data provided need some more processing to get accurate and complete knowledge. He explained information extraction types, methods and symbolic techniques. He also explained the machine learning issues. According to the paper machine learning methods for the information extraction are already classified examples. According to these learning set, system has to predict for the unseen examples. He also explained unsupervised machine learning because the machine learning disadvantage is that it needs a huge set of learning pattern and that make the entire process very time consuming and complicated. It also takes lots of manual work for the machine learning. Due to these issues unsupervised machine learning is of high importance for the information extraction in business intelligence systems. The paper explained that the real unsupervised machine learning is clustering and it works on the smallest case and the algorithms are design. Clustering is the statistical method and it automatically group data into cluster or groups. And then it replace with similar objects. Expansion is another machine learning method and it belong to the weekly supervised machine learning. In the paper he also explained some other method for machine learning e.g., self- learning, co-learning and active learning. In the end the paper explained how information can be extracted in business intelligence. Analyzing large amount of data for the purpose of decision making and to compete the organization in the marketplace. BI depends on the data warehouse and data warehouse is designed by the support of mature ETL process. When all the data is transformed into the structured form in data warehouse then all kind of data mining algorithms can be apply.

All the information provided by the BI is not useful for every department. The information varies from department to department and they pick the information according to their need and according to their strategic need. Information extraction from text base or unstructured data is one step in BI.

Sanjay et. al., [8] defined the knowledge management from Business Intelligent systems. They explained that there are lot of organizations that implement BI systems for dynamically creating and managing information that enables real time response. And that response plays a major role in decision making for enterprises. The study describe that the BI system are heart of organization and it needs to optimize. The paper focuses on the importance that the information provided by BI need to be converted in to Knowledge so that the decision can be easily taken by the higher management. The organization often has the process to convert the data in to information and then this information helps in decision making but this paper is about the automatically managing of metadata knowledge through BI system. They focuses on knowledge base process to simulate business seniors and performance matrices with critical functions to built meta data knowledge from the da ta provided by the data warehouse. In this paper the key functionalities of BI tools are explained that helps in decision making for higher management. In this paper Davenport indentified three steps for Enterprise System data transformation process 
for knowledge creation and result using for decision making. In the first phase which includes are used for the organization policies and procedures. In the second step the data is transformed when the data is extracted and analyzed and used for decision making for the enterprise. In the third step the results appeared after the decision taken by the business decision maker in the form of major changes, new actions and financial growth. These investigate how the how the business intelligence process incorporated in the transformation of enterprise systems to generate meta knowledge to get the benefits. Mathranis go through two case studies for this research, one was the Bevon practice and the second was the Cevon practices. The research focuses on the importance of Meta data knowledge from the BI system and suggest that there should be BI process which helps in providing Meta data knowledge. The will help in automatically creating knowledge from the BI application rather than getting information from the stored data and then manually converting that information into knowledge.

Boero et. al., [9] researched on CSI-Piemonte (Consortium for Information Systems in Piedmont) develops information systems for public authorities in Italy in the Piedmont region. They platform using SAS and SAP Business Object solutions. They develop the system develop a BI using SAS because the company is associated with SAS for more than 30 years and produced optimal solution for their problem and organization requirements. In this research paper software platforms are build on the basis of SAS 9.2 and SAP Business Object XI. hardware and For Grid platform SAS components are used. On the basis of these technologies BI applications are built which is used for high level of decision making. The design facilitates Data Integration, Data Quality, Data Mining, other Analytics, Querying and Reporting and Master data management tools. During the research it is proved that SAS, Dataflux and SAP Business Object BI tools are of high importance in development of BI platform. The paper is case study on the CSI-Piemonte so it explains all the components and process used from the past so many years the organization is using SAS platform for its solutions and it provides solution in a wide variety of areas. The consortium sued SAS solution to develop the largest part of regional decision making Information system. In the system BI main activities are; It provides the definition for the decision making architecture for the public body. It provide definition for data warehouse (meta data and alphanumeric data), Analysis, design and implementation activities on data warehouse. It provides Collection of analysis data from the question paper or from the internet. It provides Extraction of alphanumeric data from administrative, operational and technical archives that will be used for decision making. It provides Automatic data processing activities e.g., profiling, validation, standardization, record matching and cleansing. It provides management of data warehouse database. It also provides business intelligence services like reporting, statistical analysis, static and dynamic query and reporting. The solution also provides the expenditure flow for the organization e.g., who make the purchase, the thing are purchased, why the things are purchased, from which vendor is purchasing is done and the amount spent. Finally the implementation gave several automated solution to the organization and the gain lot of benefit from the project. So these tools can be used by any enterprise in the future.

Tangjun et. al., [10] explained the importance of ETL tools in the development of BI projects. During the ETL process vast amount of organization data is manipulated and store in data warehouse, which further help in BI application to support in decision making. In this way organization earn profit by using customer data and check their past behavior with the organization.

The paper explains how the data is extracted, transformed and load in data warehouse. The paper shows that ETL has clear module to understand and strong 
ability to do the process. The modules of ETL are easy to manage. In this paper for ETL process the techniques some techniques are used; . Optimization solution for the process is also proposed by which ETL project can be quickly built. The paper also explained the manual coding and the use of ETL tool to build the data ware house. The ETL tool provide more flexibility, efficiency, less complexity, short development cycle, less work load than the manual coding for ETL but price is high than the manual coding. The paper showed ETL tool is easy, good to use, to develop ETL.

Jayanthi Ranjan [11] explains the components, concepts techniques and benefits of the BI projects in this research paper. He writes that there are companies who are dealing with large amount of data and large amount data is manipulated in the organization e.g., customer data, call center data and cell phone user data. This data is also increasing by the time. So there are lots of challenges to manage the huge amount of data and to integrate it in the enterprise application. So for this purpose many organizations use BI application to know from the past data and to predict for the future. The goad defined by the organization can only achieve by implementing BI application. The paper explains concepts, components of BI, benefits of BI, factors influencing BI and technologies used for BI. To be on the top major industries are focusing on BI applications because due to the use of BI the companies can play with the large amount of data, analysis on the data, take knowledge from the past data and can predict for the feature behavior. The paper describes the importance of BI application and suggests that there is need for further research in exploring concepts of BI, components and benefits of BI and technologies used for BI.

Azmi et. al., [12] describe the concepts, issues and current systems of Business Intelligence. According to the paper in the last ten years Business Intelligence gained much familiarity in enterprises. Organizations feel the need for BI application in their system to get accurate information from the large data bank and then take necessary decisions. In this way, they can be dominated in current advanced IT era. The BI applications are based on fact based knowledge to improve business understanding and to get benefits from the systems. But many organizations are still confusing the concepts of BI system and they are not aware about the real benefits of BI. So many organizations are not implementing BI in their system. This paper focuses on the real benefits, drawbacks, issue, components and current system and open the way to those organization not ready for BI. This paper also discusses the BI from different point of views, the basic concepts and the idea behind the BI application and system. The paper gives detail hierarchical components of Business Intelligent system. The paper also discusses the architecture of BI system. It also gives detail benefits and issues for the organization not ready for BI environment can make some decision for BI system. Finally the paper presents the most common definition of BI found in the literature. The paper explains the components like data warehouse, repositories and the reporting as well. Although some researcher writes papers toward the readiness issues and problem for BI system but this paper explain readiness for BI system in better way. So now the organization can take better decision for BI systems.

Jui-Yu Wu [13] described that enterprise executive take decisions on the basis of data provided by the different sources like enterprises. In BI lots of tools have been developed to support the enterprise manager in decision making. But the tools are designed according to the requirements and have some limitation. Some tools developed for small scale business and some are developed for large scale business like telecom subscribers. Some tools have less data analysis capabilities and reporting limitation. But in this paper efficient data mining tools, BI framework and computation intelligence techniques have been introduced for the large scale 
organizations. The research was done on neural-network computing and also used optimizer tool for better results. In this research other tools like predictor tool, classifier tool and optimizer tool. The paper explained the BI components like; OLAP, Data Mining CRM, Database Mining, Decision Support System, Knowledge Management, Geographic Information System and Visualization. Every component explained in a better way. The paper explained the concept and importance computation intelligence base Business Intelligent system. The system includes predictor, classifier and optimizer which play a vital role in BI system. Further studies requires for computational based Business intelligent system. References [16-59] reviewed different techniques in different domains and reported their critical evaluations.

\section{Summary}

In this reviewed research paper, several tools used for decision making in term of Business Intelligence are analyzed. He explained the importance of BI tools in decision making. He explained the large amount of data used by different tools for data integration and analysis purpose for decision making by the organization. The paper mainly focused on the importance of BI system and the architecture of Online Analytical Processing (OLAP) in respect of decision support application. $\mathrm{He}$ explained that OLAP system provide analyst and manager to improve the performance of company. The paper explained different class of OLAP system e.g., ROLAP, MOLAP, HOLAP system. Every class has different purpose and user. Like ROLAP system is used for multidimensional data in data warehouse using the star or snowflake schema. This scheme is used when there is large amount of data and duplication is available. MOLAP system is used for multidimensional data in multidimensional database. Multidimensional database can be multidimensional matrices. The paper is very important in term of OLAP architecture and can be further studies.

\section{Conclusion}

BI system should be used because it help the executive in decision making. BI system also helps in business understanding and performance of business in marketplace. This paper mainly focused on process, methodologies and technologies used for business performance management. It also explained the relationship between business performance management and Business Intelligence. To manage the business performance is very difficult and critical task and it not only helps in getting maximum profit but also is very important in this fast growing IT era. Business Performance Management desired goals set by the organization.

\section{References}

[1] V. Ciobanu, F. Pop, D. Popescu and V. Cristea, "A Distributed Approach to Business Intelligence Systems Synchronization", InSymbolic and Numeric Algorithms for Scientific Computing (SYNASC), 2010 12th International Symposium on IEEE, September (2010), pp. 581-585.

[2] H. You, "A Knowledge Management Approach for Real-Time Business Intelligence. In Intelligent Systems and Applications (ISA), 2010 2nd International Workshop on IEEE, (2010) May, pp. 1-4.

[3] Z. Ren, Building Business Intelligence Application with SAP BI. In Management and Service Science, 2009. MASS'09. International Conference on IEEE, (2009) September, pp. 1-4.

[4] H. Al-Aqrabi, L. Liu, R. Hill, Z. Ding and N. Antonopoulos, "Business Intelligence Security on the Clouds: Challenges", Solutions and Future Directions. In SOSE, (2013), pp. 137-144.

[5] F. Wang, A. Yan and L. Yang, "Combined application of cloud computation technology and Business Intelligence", In Electrical and Control Engineering (ICECE), 2011 International Conference on IEEE, September (2011), pp. 2933-2936. 
[6] U. Dayal, M. Castellanos, A. Simitsis and K. Wilkinson, "Data integration flows for business intelligence", In Proceedings of the 12th International Conference on Extending Database Technology: Advances in Database Technology, Acm, March (2009), pp. 1-11.

[7] Cvitas, A. Information extraction in business intelligence systems. In MIPRO, 2010 Proceedings of the 33rd International Convention IEEE (2010), May, pp. 1278-1282.

[8] S. Mathrani, and A. Mathrani, "Leveraging Business Intelligence to Build Meta-knowledge", In System Sciences (HICSS), 2013 46th Hawaii International Conference on, IEEE, January (2013), pp. 3787-3796.

[9] M. Boero and M. E. Jackson, "Setting up, managing and using a complex Business Intelligence platform. In MIPRO", 2012 Proceedings of the 35th International Convention, IEEE, May (2012), pp. 1624-1628.

[10] T. Jun, C. Kai, F. Yu and T. Gang, "The Research \& Application of ETL Tool in Business Intelligence Project. In Information Technology and Applications, 2009. IFITA'09. International Forum on, IEEE, vol. 2, May (2009), pp. 620-623.

[11] J. Ranjan, "Business intelligence: concepts, components, techniques and benefits", Journal of Theoretical and Applied Information Technology, vol. 9, no 1, (2009), pp. 60-70.

[12] A. A. Mohamadina, M. R. B. Ghazali, M. R. B. Ibrahim and M. A. Harbawi, "Business Intelligence: Concepts, Issues and Current Systems", In Advanced Computer Science Applications and Technologies (ACSAT), 2012 International Conference on IEEE, (2012) November, pp. 234-237.

[13] J. Y. Wu, "Computational intelligence-based intelligent business intelligence system: concept and framework", In Computer and Network Technology (ICCNT), 2010 Second International Conference on, IEEE, (2010) April, pp. 334-338.

[14] M. Pirnau and C. P. Botezatu, "General information on business Intelligence and OLAP systems architecture", In Computer and Automation Engineering (ICCAE), 2010 The 2nd International Conference on, IEEE, vol. 2, (2010) February, pp. 294-297.

[15] Y. Shi and X. Lu, "The Role of Business Intelligence in Business Performance Management", In Information Management, Innovation Management and Industrial Engineering (ICIII), 2010 International Conference on, IEEE, vol. 4, (2010) November, pp. 184-186.

[16] S. Iqbal, M. Khalid and M. N. A. Khan, "A Distinctive Suite of Performance Metrics for Software Design", International Journal of Software Engineering \& Its Applications, vol. 7, no. 5, (2013).

[17] S. Iqbal and M. N. A. Khan, "Yet another Set of Requirement Metrics for Software Projects", International Journal of Software Engineering \& Its Applications, vol. 6, no. 1, (2012).

[18] M. Faizan, S. Ulhaq and M. N. A. Khan, "Defect Prevention and Process Improvement Methodology for Outsourced Software Projects", Middle-East Journal of Scientific Research, vol. 19, no. 5, (2014), pp. 674-682.

[19] M. Faizan, M. N.A. Khan and S. Ulhaq, "Contemporary Trends in Defect Prevention”, A Survey Report. International Journal of Modern Education \& Computer Science, vol. 4, no. 3, (2012).

[20] K. Khan, A. Khan, M. Aamir and M. N. A. Khan, "Quality Assurance Assessment in Global Software Development" World Applied Sciences Journal, vol. 24, no. 11, (2013).

[21] M. Amir, K. Khan, A. Khan and M. N. A. Khan, "An Appraisal of Agile Software Development Process", International Journal of Advanced Science \& Technology, vol. 58, (2013).

[22] M. Khan and M. N. A. Khan, "Exploring Query Optimization Techniques in Relational Databases", International Journal of Database Theory \& Application, vol. 6, no. 3, (2013).

[23] M. N. A. Khan, M. Khalid and S. UlHaq, "Review of Requirements Management Issues in Software Development", International Journal of Modern Education \& Computer Science, vol. 5, no. 1, (2013).

[24] M. Umar and M. N. A. Khan, "A Framework to Separate NonFunctional Requirements for System Maintainability", Kuwait Journal of Science \& Engineering, vol. 39, no. 1 B, (2012), pp. 211- 231.

[25] M. Umar and M. N. A. Khan, "Analyzing Non-Functional Requirements (NFRs) for software development", In IEEE 2nd International Conference on Software Engineering and Service Science (ICSESS), (2011), pp. 675-678.

[26] M. N. A. Khan, C. R. Chatwin and R. C. Young, "A framework for post-event timeline reconstruction using neural networks", digital investigation, vol. 4, no. 3, (2007), pp. 146-157.

[27] M. N. A. Khan, C. R. Chatwin and R. C. Young, "Extracting Evidence from File system Activity using Bayesian Networks", International journal of Forensic computer science, vol. 1, (2007), pp. 50-63.

[28] M. N. A. Khan, "Performance analysis of Bayesian networks and neural networks in classification of file system activities", Computers \& Security, vol. 31, no. 4, (2012), pp. 391-401.

[29] M. Rafique and M. N. A. Khan, "Exploring Static and Live Digital Forensics: Methods, Practices and Tools", International Journal of Scientific \& Engineering Research, vol. 4, no. 10, (2013), pp. 1048-1056.

[30] M. S. Bashir and M. N. A. Khan, "Triage in Live Digital Forensic Analysis", International journal of Forensic Computer Science, vol. 1, (2013), pp. 35-44.

[31] A. Sarwar and M. N. A. Khan, "A Review of Trust Aspects in Cloud Computing Security", International Journal of Cloud Computing and Services Science (IJCLOSER), vol. 2, no. 2, (2013), pp. 116-122.

[32] A. H. Gondal and M. N. A. Khan, "A review of fully automated techniques for brain tumor detection from MR images", International Journal of Modern Education and Computer Science (IJMECS), vol. 5, no. 2, (2013), pp. 55. 
[33] A. Zia and M. N. A. Khan, "Identifying key challenges in performance issues in cloud computing", International Journal of Modern Education and Computer Science (IJMECS), vol. 4, no. 10, (2012), pp. 59.

[34] K. U. Rehman and M. N. A. Khan, "The Foremost Guidelines for Achieving Higher Ranking in Search Results through Search Engine Optimization", International Journal of Advanced Science and Technology, vol. 52, (2013), pp. 101-110.

[35] M. Khan and M. N. A. Khan, "Exploring query optimization techniques in relational databases", International Journal of Database Theory \& Application, vol. 6, no. 3, (2013).

[36] R. Shehzad, M. N. KHAN and M. Naeem, "Integrating knowledge management with business intelligence processes for enhanced organizational learning", International Journal of Software Engineering and Its Applications, vol. 7, no. 2, (2013), pp. 83-91.

[37] S. U. Haq, M. Raza, A. Zia and M. N. A. Khan, "Issues in global software development: A critical review", Journal of Software Engineering and Applications, vol. 4, no. 10, (2015), pp. 590.

[38] A. S. Shah, M. N. A. Khan and A. Shah, "An appraisal of off-line signature verification techniques", International Journal of Modern Education and Computer Science, vol. 7, no. 4, (2015), pp. 67-75.

[39] A. Zia and M. N. A. Khan, "A Scheme to Reduce Response Time in Cloud Computing Environment", International Journal of Modern Education and Computer Science (IJMECS), vol. 5, no. 6, (2013), pp. 56

[40] M. Tariq and M. N. A. Khan, "The Context of Global Software Development: Challenges, Best Practices and Benefits", Information Management \& Business Review, vol. 3, no. 4, (2011).

[41] A. Shahzad, M. Hussain and M. N. A. Khan, "Protecting from Zero-Day Malware Attacks", Middle-East Journal of Scientific Research, vol. 17, no. 4, (2013), pp. 455-464.

[42] A. A. Khan and M. Khan, "Internet content regulation framework", International Journal of U-\& EService, Science \& Technology, vol. 4, no. 3, (2011).

[43] K. Ullah and M. N. A. Khan, "Security and Privacy Issues in Cloud Computing Environment: A Survey Paper", International Journal of Grid and Distributed Computing, vol. 7, no. 2, (2014), pp. 89-98.

[44] A. A. Abbasi, M. N. A. Khan and S. A. Khan, "A Critical Survey of Iris Based Recognition Systems", Middle-East Journal of Scientific Research, vol. 15, no. 5, (2013), pp. 663- 668.

[45] M. N. A. Khan, S. A. Qureshi and N. Riaz, "Gender classification with decision trees", Int. J. Signal Process. Image Process. Patt. Recog, vol. 6, (2013), pp. 165-176.

[46] S. S. Ali and M. N. A. Khan, "ICT Infrastructure Framework for Microfinance Institutions and Banks in Pakistan: An Optimized Approach", International Journal of Online Marketing (IJOM), vol. 3, no. 2, (2013), pp. 75-86.

[47] A. Mahmood, M. Ibrahim and M. N. A. Khan, "Service Composition in the Context of Service Oriented Architecture", Middle East Journal of Scientific Research, vol. 15, no. 11, (2013).

[48] M. A. Masood and M. N. A. Khan, "Clustering Techniques in Bioinformatics", I. J. Modern Education and Computer Science, vol. 1, (2015), pp. 38-46.

[49] T. Ur Rehman, M. N. A. Khan and N. Riaz, "Analysis of Requirement Engineering Processes, Tools/Techniques and Methodologies", International Journal of Information Technology and Computer Science (IJITCS), vol. 5, no. 3, (2013), pp. 40.

[50] R. Ahmed and M. N. A. Khan, "An Analytical Review of Stereovision Techniques to Reconstruct 3D Coordinates", International Journal of Information Technology and Computer Science (IJITCS), vol. 5, no. 7, (2013), pp. 80.

[51] M. F. Malik and M. N. A. Khan, "An Analysis of Performance Testing in Distributed Software Applications", International Journal of Modern Education \& Computer Science, vol. 8, no. 7, (2016).

[52] R. Naz, M. N. A. Khan and M. Aamir, "Scrum-Based Methodology for Product Maintenance and Support", IJEM-International Journal of Engineering and Manufacturing (IJEM), vol. 6, no. 1, (2016), pp. 10.

[53] S. Rahman and M. N. A. Khan, "Digital Forensics through Application Behavior Analysis", International Journal of Modern Education \& Computer Science, (2016).

[54] S. Shafqat, M. N. A Khan, N. Riaz and K. Khan, "Identity Matrix: Architecture Framework for Trusted Cloud Computing through Cloud Intellect", Journal of Internet Technology, vol. 17, no. 4, (2016), pp. 2.

[55] G. Ahmed, M. N. A. Khan and M. S. Bashir, "A Linux-based IDPS using Snort", Computer Fraud \& Security, vol. 2015, no. 8, (2015), pp. 13-18.

[56] M. Rafique and M. N. A. Khan, "Profiling software applications for forensic analysis", Computer Fraud \& Security, vol. 2015, no. 6, (2015), pp. 13-18.

[57] M. Khan, M. Akram and N. Riaz, "A comparative analysis of software protection schemes", Int. Arab J. Inf. Technol., vol. 12, no. 3, (2015), pp. 286-295.

[58] G. Ahmed, M. Hussain and M. N. A. Khan, "Characterizing Strengths of Snort-based IDPS", Research Journal of Recent Sciences, (2014).

[59] A. Abbasi and M. Khan, "Iris-Pupil Thickness Based Method for Determining Age Group of a Person", International Arab Journal of Information Technology, no. 6, (2016) November. 